\title{
Characteristic Formulae for Relations with Nested Fixed Points*
}

\author{
Luca Aceto Anna Ingólfsdóttir ${ }^{\dagger}$ \\ ICE-TCS, School of Computer Science \\ Reykjavik University \\ Reykjavik, Iceland \\ \{luca, annai\}@ru.is
}

\begin{abstract}
A general framework for the connection between characteristic formulae and behavioral semantics is described in [2]. This approach does not suitably cover semantics defined by nested fixed points, such as the $n$-nested simulation semantics for $n$ greater than 2 . In this study we address this deficiency and give a description of nested fixed points that extends the approach for single fixed points in an intuitive and comprehensive way.
\end{abstract}

\section{Introduction}

In process theory it has become a standard practice to describe behavioural semantics in terms of equivalences or preorders. A wealth of such relations has been classified by van Glabbeek in his linear time/branching time spectrum [4]. Branching-time behavioural semantics are often defined as largest fixed points of monotonic functions over the complete lattice of binary relations over processes.

In [2] we give a general framework to reason about how this type of behavioral semantics can be characterized by a modal logic equipped with a greatest fixed point operator, or more precisely by characteristic formulae expressed in such a logic. In that reference we show that a behavioural relation that is derived as a greatest fixed point of a function of relations over processes is given by the greatest fixed point of the semantic interpretation of a logical declaration that expresses the function in a formal sense that is defined in present paper. Roughly speaking if a logical declaration describes a monotonic function over a complete lattice then its fixed point describes exactly the fixed point of the function. In [2] preorders and equivalences such as simulation preorder and bisimulation equivalence are characterized following this approach in a simple and constructive way. However, when the definition of a behavioural relation involves nested fixed points, i. e. when the monotonic function that defines the relation takes another fixed point as an argument, things get more complicated. The framework offered in [2] only deals with nesting on two levels and in a rather clumsy and unintuitive way. Furthermore it does not extend naturally to deeper nesting, like for the $n$-nested simulations for $n>2$. In this study we address this deficiency and define a logical framework in which relations obtained as a chain of nested fixed points of monotonic functions can be characterized following general principles. This extends the approach for single fixed points in an intuitive and comprehensive way.

As the applications we present in the paper only deal with nesting of greatest fixed points, this study only focuses on greatest fixed points. However it is straightforward to extend it to deal with alternating nesting of both least and greatest fixed points. We also believe that our approach gives some idea about how fixed point theories in different domains can be compared in a structured way.

\footnotetext{
*Supported by the project Processes and Modal Logics' (project nr. 100048021) of the Icelandic Research Fund.

$\dagger$ Supported by the VELUX visiting professorship funded by the VILLUM FOUNDATION.
} 
The remainder of the paper is organized as follows. Section 2 presents some background on fixed points of monotone functions. Section 3 briefly introduces the model of labelled transition systems and some results on behavioural relations defined as greatest fixed points of monotonic functions over binary relations. The logic we shall use to define characteristic formulae in a uniform fashion is discussed in Section 4. The key notion of a declaration expressing a monotone function is also given in that section. Section 5 is devoted to an application of our framework to the logical characterization of the family of nested simulation semantics.

\section{Posets, monotone functions and fixed points}

In this section we introduce some basic concepts we need in the paper.

\section{Definition 2.1}

- A partially ordered set, or poset, $\left(A, \sqsubseteq_{A}\right)$ (usually referred to simply as $A$ ) consists of a set $A$ and a partial order $\sqsubseteq_{A}$ over it.

- If $A$ is a poset and $M \subseteq A$, then $a \in A$ is an upper bound for $M$ if $m \sqsubseteq_{A}$ a for all $m \in M$. a is $a$ least upper bound (lub) for $M$ if it is an upper bound for $M$ and if whenever $b$ is an upper bound for $M$ then $a \sqsubseteq_{A} b$.

- A poset $A$ is a complete lattice if the lub for $M$ exists for all $M \subseteq A$.

- For posets $A$ and $B$, a function $\phi: A \rightarrow B$ is monotone if it is order preserving; it is an isomorphism if it is bijective and both $\phi$ and its inverse $\phi^{-1}$ are monotone. We let $A \rightarrow$ mono $B$ denote the set of monotone functions from $A$ to $B$.

- If $A$ is a poset and $f \in A \rightarrow$ mono $A$, then $x \in A$ is a fixed point of $f$ if $f(x)=x$. We write $v f$ (or $v x . f(x))$ for the greatest fixed point of $f$ if it exists.

- If $A$ and $B$ are posets, $f \in A \rightarrow_{\text {mono }} A$ and $\phi \in A \rightarrow_{\text {mono }} B$ is an isomorphism then we define $\phi^{*} f: B \rightarrow B$ as $\phi^{*} f=\phi \circ f \circ \phi^{-1}$.

Note that the $l u b$ of a subset of a poset $A$ is unique if it exists and the same holds for greatest fixed points of monotone functions over posets. It is well known, that if $A$ and $B$ are posets/complete lattices and $I$ is some set, then the Cartesian product $A \times B$ and the function space $I \rightarrow A$ are a posets/complete lattices under the pointwise ordering. The following theorem is due to Tarski.

Theorem 2.2 ([[10]) If $A$ is a complete lattice and $f \in A \rightarrow_{\text {mono }} A$, then $f$ has a unique greatest fixed point.

The theorem below is proved in [2] and is the key to the general theory we present in this paper.

Theorem 2.3 Let $A$ and $B$ be posets, $f \in A \rightarrow_{\text {mono }} A$ and $\phi: A \rightarrow B$ be an isomorphism. Then $v f$ exists iff $v\left(\phi^{*} f\right)$ exists. If these fixed points exist then $\phi(v f)=v\left(\phi^{*} f\right)$.

\section{Labelled transition systems and behavioural relations}

It has become standard practice to describe behavioural semantics of processes by means of a labelled transition system as defined below.

Definition 3.1 ([|7]) A labelled transition system (LTS) is a triple $P=(\mathbf{P}, \mathbf{A}, \rightarrow)$ where

- A is a finite set (of actions), 
- $\mathbf{P}$ is a finite set (of processes), and

- $\rightarrow \subseteq \mathbf{P} \times \mathbf{A} \times \mathbf{P}$ is a transition relation.

As usual, we write $p \stackrel{a}{\longrightarrow} p^{\prime}$ for $\left(p, a, p^{\prime}\right) \in \rightarrow$. Throughout this paper we assume that the set $\mathbf{A}$ is fixed.

As LTSs are in general to concrete, processes are compared by preorders or equivalences. These are often obtained as the greatest fixed points to monotone endofunctions on the complete lattice $\mathscr{P}(\mathbf{P} \times \mathbf{P})$. We will show some example of such functions but first we state and prove some properties.

Definition 3.2 If $\mathscr{F} \in \mathscr{P}(\mathbf{P} \times \mathbf{P}) \rightarrow_{\text {mono }} \mathscr{P}(\mathbf{P} \times \mathbf{P})$ and $A \in \mathscr{P}(\mathbf{P} \times \mathbf{P})$, we define

- $\tilde{\mathscr{F}}: S \mapsto\left(\mathscr{F}\left(S^{-1}\right)\right)^{-1}$, and

- $\mathscr{F} \cap A: S \mapsto \mathscr{F}(S) \cap A$.

The following lemma will be applied below.

Lemma 3.3 Let $\mathscr{F} \in \mathscr{P}(\mathbf{P} \times \mathbf{P}) \rightarrow_{\text {mono }} \mathscr{P}(\mathbf{P} \times \mathbf{P})$ and $A \in \mathscr{P}(\mathbf{P} \times \mathbf{P})$. Then

- $\tilde{\mathscr{F}}, \mathscr{F} \cap A \in \mathscr{P}(\mathbf{P} \times \mathbf{P}) \rightarrow_{\text {mопо }} \mathscr{P}(\mathbf{P} \times \mathbf{P})$,

- $\boldsymbol{v} \tilde{\mathscr{F}}=(\boldsymbol{v} \mathscr{F})^{-1}$ and

- $\widetilde{\mathscr{F} \cap A}=\tilde{\mathscr{F}} \cap A^{-1}$.

Proof The first two statements are proved in [2]. To prove the third one we proceed follows:

$$
(\widetilde{\mathscr{F} \cap A})(S)=\left((\mathscr{F} \cap A)\left(S^{-1}\right)\right)^{-1}=\left(\mathscr{F}\left(S^{-1}\right)\right)^{-1} \cap A^{-1}=\left(\tilde{\mathscr{F}} \cap A^{-1}\right)(S) .
$$

We will complete this section by giving some examples of endofunction that define some standard behavioural preorders and equivalences [4, 1].

Definition 3.4 Let $\mathscr{F}: \mathscr{P}(\mathbf{P} \times \mathbf{P}) \rightarrow \mathscr{P}(\mathbf{P} \times \mathbf{P})$ be defined as follows:

$$
(p, q) \in \mathscr{F}(S) \text { iff } \forall a \in \mathbf{A}, p^{\prime} \in \mathbf{P} . p \stackrel{a}{\longrightarrow} p^{\prime} \Rightarrow \exists q^{\prime} \in \mathbf{P} . q \stackrel{a}{\longrightarrow} q^{\prime} \wedge\left(p^{\prime}, q^{\prime}\right) \in S .
$$

It is easy to check that $\mathscr{F}$ is monotonic and therefore it has a greatest fixed point.

Definition 3.5 We define:

- $\mathscr{F}_{\text {sim }}=\mathscr{F}$ and $\sqsubseteq_{\text {sim }}=v \mathscr{F}_{\text {sim }}$ (simulation preorder),

- $\mathscr{F}_{\text {opsim }}=\tilde{\mathscr{F}}$ and $\sqsubseteq_{\text {opsim }}=v \mathscr{F}_{\text {opsim }}$ (inverse simulation preorder),

- $\sim_{\text {sim }}=\sqsubseteq_{\operatorname{sim}} \cap \sqsubseteq_{\text {opsim }}$ (simulation equivalence) and

- $\mathscr{F}_{\text {bisim }}=\mathscr{F}_{\text {sim }} \cap \mathscr{F}_{\text {opsim }}$ and $\sim_{\text {bisim }}=\boldsymbol{v} \mathscr{F}_{\text {bisim }}$ (bisimulation equivalence).

\section{Equational modal $v$-calculi with nested fixed-points}

In this section we introduce variants of the standard equational modal $\mu$-calculus [8]. Like in [9] these variants only allow for nested fixed points, i. e. where the logical languages form a hierarchy where fixed points in a language on one level are allowed as constants in the logic on the level above. Our approach, however, differs from the original one in the sense that the fixed-point operator is explicit in the syntax and can therefore be used in logical expressions. In this study we only focus on greatest fixed points (which explains the title of this section) but the framework can easily be extended to involve nesting 
of both greatest and least fixed points. The logical languages we introduce depend on the implicitly assumed fixed finite set $\mathbf{A}$.

Our basic logic $\mathscr{M}$ is the standard Hennessy-Milner Logic (HML) [6] without variables. This logic is generated by $\Sigma=\left(\Sigma_{0}, \Sigma_{1}, \Sigma_{2}\right)$ where $\Sigma_{0}=\{t t, f f\}$ are the constants or the operators of arity $0, \Sigma_{1}=$ $\{\langle a\rangle,[a], a \in \mathbf{A}\}$ are the operators of arity 1 , and $\Sigma_{2}=\{\wedge, \vee\}$ are the operators of arity 2.

The formulae in $\mathscr{M}$ are interpreted over an LTS $(\mathbf{P}, \mathbf{A}, \rightarrow)$ as the set of elements from $\mathbf{P}$ that satisfy them. Satisfaction is determined by a semantic function that is defined below. For $M \subseteq \mathbf{P}$ we let $\langle\cdot a \cdot\rangle M=$ $\{p \in \mathbf{P} \mid \exists q \in M \cdot p \stackrel{a}{\longrightarrow} q\}$, and $[\cdot a \cdot] M=\overline{\langle\cdot a \cdot\rangle \bar{M}}$ where $\bar{M}$ is the complement of the set $M$.

Definition 4.1 The semantic function $\mathscr{M}[[]]$ is defined as follows:

1. $\mathscr{M}[[t t]]=\mathbf{P}, \mathscr{M}[[f f]]=\emptyset$,

2. $\mathscr{M}\left[\left[F_{1} \wedge F_{2}\right]\right]=\mathscr{M}\left[\left[F_{1}\right]\right] \cap \mathscr{M}\left[\left[F_{2}\right]\right], \mathscr{M}\left[\left[F_{1} \vee F_{2}\right]\right]=\mathscr{M}\left[\left[F_{1}\right]\right] \cup \mathscr{M}\left[\left[F_{2}\right]\right]$,

3. $\mathscr{M}[[\langle a\rangle F]=\langle\cdot a \cdot\rangle \mathscr{M}[[F]], \mathscr{M}[[[a] F]]=[\cdot a \cdot] \mathscr{M}[[F]]$.

The logic $\mathscr{V}$ is the standard Hennessy-Milner logic with variables that was introduced in [9]. It assumes a finite index set $I$ and an $I$-indexed set of variables $\mathscr{X}$. In what remains of this paper we assume a fixed pair of such $I$ and $\mathscr{X}$, unless stated otherwise.

As the elements of $\mathscr{V}$ typically contain variables, they have to be interpreted with respect to a variable interpretation $\sigma \in \mathscr{P}(\mathbf{P})^{I}$ that associates to each $i \in I$ the set of processes in $\mathbf{P}$ that are assumed to satisfy the variable $X_{i}$. The semantic function $\mathscr{V}[[]]$ in this case takes a formula $F$ and a $\sigma \in \mathscr{P}(\mathbf{P})^{I}$ and delivers an element of $\mathscr{P}(\mathbf{P})$.

Definition 4.2 The semantic function $\mathscr{V}[[]]$ is defined as follows:

1. $\mathscr{V}[[F]] \sigma=\mathscr{M}[[F]]$ if $F \in \Sigma_{0}$,

2. $\mathscr{V}\left[\left[X_{i}\right] \sigma=\sigma(i), i \in I\right.$,

3. $\left.\mathscr{V}\left[\left[F_{1} \wedge F_{2}\right]\right] \sigma=\mathscr{V} \llbracket\left[F_{1}\right]\right] \sigma \cap \mathscr{V}\left[\left[F_{2}\right]\right] \sigma, \mathscr{V}\left[\left[F_{1} \vee F_{2}\right]\right] \sigma=\mathscr{V}\left[\left[F_{1}\right]\right] \sigma \cup \mathscr{V}\left[\left[F_{2}\right]\right] \sigma$,

4. $\mathscr{V} \llbracket[\langle a\rangle F] \sigma=\langle\cdot a \cdot\rangle \mathscr{V}[[F]] \sigma, \mathscr{V}[[[a] F] \sigma=[\cdot a \cdot] \mathscr{V}[[F]] \sigma$.

In [9] the meaning of the variables in the logic $\mathscr{V}$ is defined by means of a declaration, or a function $D: I \rightarrow \mathscr{V}$. Intuitively the syntactic function generates a monotonic endofunction $\mathscr{V}[[D]]$ over $\mathscr{P}(\mathbf{P})^{I}$ defined by $(\mathscr{V}[[D]])(i)=\mathscr{V}[[D(i)]]$ for all $i \in I$. By Theorem $2.2, \mathscr{V}[[D]]$ has a unique largest fixed point $v \mathscr{V}[[D]] \in \mathscr{P}(\mathbf{P})^{I}$ that can be used to give the semantics for the variables and the formulae that contain those in the logic $\mathscr{V}$. We can then use this to extend the logic $\mathscr{M}$ with $\{v D(i) \mid i \in I\}$ as constants interpreted as $\{v \mathscr{V}[[D]](i) \mid i \in I\}$. By this we get a logic $\mathscr{M}^{\prime}$ that is generated by $\Sigma^{\prime}=\left(\Sigma_{0} \cup\{v D(i) \mid i \in\right.$ $\left.I\}, \Sigma_{2}, \Sigma_{3}\right)$. Then this procedure can be repeated for another declaration that possibly depends on $v D$ as a constant and with $\mathscr{M}^{\prime}$ as the basic logic. The following example shows how this construction works.

Example Let $I=\{1\}, \mathscr{X}=\left\{X_{1}\right\}$ and $\mathbf{A}=\{a, b\}$ and let the property "invariantly $\langle a\rangle t t$ " be defined as the greatest fixed point corresponding to the declaration $D_{0}$ defined as $D_{0}(1)=\langle a\rangle t t \wedge[a] X_{1} \wedge[b] X_{1}$. To interpret this we define $\mathscr{M}=\mathscr{M}_{0}$ and $\mathscr{V} 0=\mathscr{V}$ where $\mathscr{M}$ and $\mathscr{V}$ have the meaning described above. The derived semantic function $\mathscr{V}_{0}\left[\left[D_{0}\right]\right]: \mathscr{P}(\mathbf{P})^{\{1\}} \rightarrow \mathscr{P}(\mathbf{P})^{\{1\}}$ is easily shown to be monotonic and has the greatest fixed point $v \mathscr{V}_{0}\left[\left[D_{0}\right] \in \mathscr{P}(\mathbf{P})^{\{1\}}\right.$. Now we define $\mathscr{M}_{1}$ as the extension of $\mathscr{M}_{0}$ that is generated by $\Sigma^{1}=\left(\left\{t t, f f, v D_{0}(1)\right\}, \Sigma_{1}, \Sigma_{2}\right)$, i.e. has $v D_{0}(1)$ as a constant that is interpreted as $v \mathscr{V}_{0}\left[\left[D_{0}\right]\right](1)$, i.e. $\mathscr{M}_{1}\left[\left[v D_{0}(1)\right]\right]=v \mathscr{V}_{0}\left[\left[D_{0}\right]\right](1)$.

Next let us assume that we have the declaration $D_{1}:\{1\} \rightarrow \mathscr{V}_{1}$ where $\mathscr{V}_{1}$ is the variable logic generated by $\left(\left\{t t, f f, v D_{0}(1), X_{1}\right\}, \Sigma_{2}, \Sigma_{3}\right)$ and $D_{1}$ is defined as $D_{1}(1)=\langle b\rangle v D_{0}(1) \wedge[b] X_{1}$. As before the declaration is interpreted over $\mathscr{P}(\mathbf{P})^{\{1\}}$ but using $\mathscr{M}_{1}[[]]$ to interpret the constant $v D_{0}(1)$. Again $D_{1}$ is interpreted by using $\mathscr{V}_{1}[[]]$ which leads to a monotonic endofunction $\mathscr{V}_{1}\left[\left[D_{1}\right]\right]$ over $\mathscr{P}(\mathbf{P})^{\{1\}}$ with a fixed point 
$v \mathscr{V}_{1}\left[\left[D_{1}\right]\right]$. The logic $\mathscr{M}_{2}$ is now defined as the one generated by $\Sigma^{2}=\left(\left\{t t, f f, v D_{1}(1), v D_{2}(1)\right\}, \Sigma_{2}, \Sigma_{3}\right)$ where $\mathscr{M}_{0}[[]]$ and $\mathscr{M}_{1}[[]]$ are used to define the meaning of $v D_{1}(1)$ and $v D_{2}(1)$ respectively.

We will now generalize this procedure and define our hierarchy of nested fixed point logics, derived from a sequence of nested declarations $D_{j}, j=1,2, \ldots, N$, i.e. where for each $n<N, D_{n+1}$ is allowed to depend on the constants $t$, ff and $v D_{j}(i)$ for $j \leq n$ and $i \in I$. In the definition we assume a finite index set $I$ and an $I$-indexed variable set $\mathscr{X}$. We use the notation $\mathscr{G}\left(\Sigma_{0}\right)$ for the logic generated by $\left(\Sigma_{0}, \Sigma_{1}, \Sigma_{2}\right)$ and $\mathscr{G}_{I}\left(\Sigma_{0}\right)$ for the logic generated by $\left(\Sigma_{0} \cup \mathscr{X}, \Sigma_{1}, \Sigma_{2}\right)$.

\section{Definition 4.3}

- Define

$-\Sigma_{0}^{0}=\{t t, f f\}$,

- $\mathscr{M}_{0}=\mathscr{G}\left(\Sigma_{0}^{0}\right)$ and

- $\mathscr{V}_{0}=\mathscr{G}_{I}\left(\Sigma_{0}^{0}\right)$.

- For $n \geq 1$, if $D_{n}: I \rightarrow \mathscr{V}_{n}$, define

- $\Sigma_{0}^{n+1}=\Sigma_{0}^{n} \cup\left\{v D_{n}(i) \mid i \in I\right\}$,

- $\mathscr{M}_{n+1}=\mathscr{G}\left(\Sigma_{0}^{n+1}\right)$ and

- $\mathscr{V}_{n+1}=\mathscr{G}_{I}\left(\Sigma_{0}^{n+1}\right)$.

To define the semantic functions associated with these logics we need the following lemma.

Lemma 4.4 Assume that $\mathscr{M}=\mathscr{G}(C)$ and $\mathscr{V}=\mathscr{G}_{I}(C)$ for some set of constants $C$ where $\mathscr{M} \llbracket[c]$ is well defined for all $c \in C$. Then for all $D: I \rightarrow \mathscr{V}$, the derived semantic function $\mathscr{V}[[D]]$ defined by

$$
\forall i \in I .(\mathscr{V}[[D]] \sigma)(i)=\mathscr{V}[[D(i)]] \sigma
$$

is in $\mathscr{P}(\mathbf{P})^{I} \rightarrow_{\text {mono }} \mathscr{P}(\mathbf{P})^{I}$ and hence, by Theorem $2.2 \vee \mathscr{V}\left[[D] \in \mathscr{P}(\mathbf{P})^{I}\right.$ exists.

Now we are ready to define the semantic functions for $\mathscr{M}_{n}$ and $\mathscr{V}_{n}$ for all $n \geq 0$.

\section{Definition 4.5}

- $\mathscr{M}_{0}=\mathscr{M}$ and $\mathscr{V}_{0}=\mathscr{V}$ as defined in Definition 4.1 and 4.2 respectively.

- For $n \geq 0$ the semantic functions for $\mathscr{M}_{n+1}$ is defined as follows:

1. $\mathscr{M}_{n+1}[[F]]=\mathscr{M}_{n}\left[[F]\right.$ if $F \in \Sigma_{0}^{n}$,

2. $\mathscr{M}_{n+1}\left[\left(v D_{n}\right)(i)\right]=v \mathscr{V}_{n}\left[\left[D_{n}\right]\right](i)$ for $i \in I$,

3. $\mathscr{M}_{n+1}\left[\left[F_{1} \wedge F_{2}\right]\right]=\mathscr{M}_{n+1}\left[\left[F_{1}\right]\right] \cap \mathscr{M}_{n+1}\left[\left[F_{2}\right]\right], \mathscr{M}_{n+1}\left[\left[F_{1} \vee F_{2}\right]\right]=\mathscr{M}_{n+1}\left[\left[F_{1}\right]\right] \cup \mathscr{M}_{n+1}\left[\left[F_{2}\right]\right]$,

4. $\mathscr{M}_{n+1}[[\langle a\rangle F]]=\langle\cdot a \cdot\rangle \mathscr{M}_{n+1}[[F]], \mathscr{M}_{n+1}[[[a] F]]=[\cdot a \cdot] \mathscr{M}_{n+1}[[F]]$.

- For $n \geq 0$ the semantic function for $\mathscr{V}_{n+1}$ is defined as follows:

1. $\mathscr{N}_{n+1}[[F]] \sigma=\mathscr{M}_{n+1}[[F]]$ if $F \in \Sigma_{n}^{0}$,

2. $\mathscr{V}_{n+1}\left[\left[X_{i}\right]\right] \sigma=\sigma(i), i \in I$,

3. $\mathscr{V}_{n+1}\left[\left[F_{1} \wedge F_{2}\right]\right] \sigma=\mathscr{V}_{n+1}\left[\left[F_{1}\right]\right] \sigma \cap \mathscr{V}_{n+1}\left[\left[F_{2}\right]\right] \sigma, \mathscr{V}_{n+1}\left[\left[F_{1} \vee F_{2}\right]\right] \sigma=\mathscr{V}_{n+1}\left[\left[F_{1}\right]\right] \sigma \cup \mathscr{V}_{n+1}\left[\left[F_{2}\right]\right] \sigma$,

4. $\mathscr{V}_{n+1}\left[[\langle a\rangle F] \sigma \sigma=\langle\cdot a \cdot\rangle \mathscr{V}_{n+1}[[F]] \sigma, \mathscr{V}_{n+1}[[[a] F]] \sigma=[\cdot a \cdot] \mathscr{V}_{n+1}[[F]] \sigma\right.$. 


\subsection{Characteristic Formulae by means of Declarations}

The aim of this section is to show how each process $p \in \mathbf{P}$ can be characterized up to a binary relation $\bowtie$ over processes (such as an equivalence or a preorder) by a single formula, the so called characteristic formula for $p$ up to $\bowtie$.

To achieve this, we take $I=\mathbf{P}$ in the definitions in the previous section. A declaration $D$ for a variable $\operatorname{logic} \mathscr{V}$ assigns exactly one formula $D(p)$ from $\mathscr{V}$ to each process $p \in \mathbf{P}$. We have seen that each such function induces an endofunction $\mathscr{V}[[D]] \in \mathscr{P}(\mathbf{P})^{\mathbf{P}} \rightarrow_{\text {mono }} \mathscr{P}(\mathbf{P})^{\mathbf{P}}$ and therefore $\mathscr{V}[[D]]$ exists. This leads to the following definition:

Definition 4.6 A declaration $D$ for the logic $\mathscr{V}$ characterizes $\bowtie \subseteq \mathbf{P} \times \mathbf{P}$ iff for each $p, q \in \mathbf{P}$,

$$
(p, q) \in \bowtie \text { iff } q \in(v \mathscr{V}[[D]])(p) .
$$

In what follows, we will describe how we can devise a characterizing declaration for a relation that is obtained as a fixed point, or a sequence of nested fixed points of monotone endofunctions, which can be expressed in the logic. In order to define this precisely we use the notation introduced in Definition 4.7 below.

Definition 4.7 If $S \subseteq \mathbf{P} \times \mathbf{P}$ we define the variable interpretation $\sigma_{S} \in \mathscr{P}(\mathbf{P})^{\mathbf{P}}$ associated to $S$ by

$$
\sigma_{S}(p)=\{q \in \mathbf{P} \mid(p, q) \in S\}, \text { for each } p \in \mathbf{P} .
$$

Thus $\sigma_{S}$ assigns to $p$ all those processes $q$ that are related to it via $S$.

Definition 4.8 A declaration $D$ for $\mathscr{V}$ expresses a monotone endofunction $\mathscr{F}$ on $\mathscr{P}(\mathbf{P} \times \mathbf{P})$ when

$$
(p, q) \in \mathscr{F}(S) \text { iff } q \in \mathscr{V}[[D(p)]] \sigma_{S}=\left(\mathscr{V}[[D]] \sigma_{S}\right)(p),
$$

for every relation $S \subseteq \mathbf{P} \times \mathbf{P}$ and every $p, q \in \mathbf{P}$.

We need the following to prove our main result.

Definition 4.9 Let $\Phi: \mathscr{P}(\mathbf{P} \times \mathbf{P}) \rightarrow \mathscr{P}(\mathbf{P})^{\mathbf{P}}$ be defined by $\Phi(S)=\sigma_{S}$.

Lemma 4.10

- $\Phi: \mathscr{P}(\mathbf{P} \times \mathbf{P}) \rightarrow \mathscr{P}(\mathbf{P})^{\mathbf{P}}$ is an isomorphism.

- If $A_{1}, A_{2} \in \mathscr{P}(\mathbf{P} \times \mathbf{P})$ and $\mathscr{F}_{1}, \mathscr{F}_{2} \in \mathscr{P}(\mathbf{P} \times \mathbf{P}) \rightarrow_{\text {mono }} \mathscr{P}(\mathbf{P} \times \mathbf{P})$ then

- $\Phi\left(A_{1} \cap A_{2}\right)=\Phi\left(A_{1}\right) \cap \Phi\left(A_{2}\right)$,

- $\Phi^{*}\left(\mathscr{F}_{1} \cap A_{1}\right)=\Phi^{*}\left(\mathscr{F}_{1}\right) \cap \Phi\left(A_{1}\right)$ and

- $\Phi^{*}\left(\mathscr{F}_{1} \cap \mathscr{F}_{2}\right)=\Phi^{*}\left(\mathscr{F}_{1}\right) \cap \Phi^{*}\left(\mathscr{F}_{2}\right)$.

Proof The first part is proved in [2] whereas the second part follows directly from the definition of $\Phi$.

Corollary 4.11 Assume that $D \in \mathbf{P} \rightarrow \mathscr{V}$ and $\mathscr{F} \in \mathscr{P}(\mathbf{P} \times \mathbf{P}) \rightarrow_{\text {mono }} \mathscr{P}(\mathbf{P} \times \mathbf{P})$. Then

$$
\text { D expresses } \mathscr{F} \text { iff } \Phi^{*}(\mathscr{F})=\mathscr{V}[[D]] \text { iff } D \text { characterizes } v \mathscr{F} \text {. }
$$

\section{Applications}

Following the approach in [2], we define declarations $D$ and $\tilde{D}$ that express the functions $\mathscr{F}$ and $\tilde{\mathscr{F}}$ that were defined in Section 3 ,

Definition 5.1 Let

- Let $D: p \mapsto \bigwedge_{a \in \mathbf{A}} \bigwedge_{p^{\prime} \in \mathbf{P} . p \stackrel{a}{\longrightarrow} p^{\prime}}\langle a\rangle X_{p^{\prime}}$ and

- $\tilde{D}: p \mapsto \bigwedge_{a \in \mathbf{A}}[a] \bigvee_{p^{\prime} \in \mathbf{P} . p \stackrel{a}{\longrightarrow} p^{\prime}} X_{p^{\prime}}$. 
From [2] we have:

\section{Lemma 5.2}

- D expresses $\mathscr{F}$ and characterizes $\boldsymbol{v} \mathscr{F}$, and

- $\tilde{D}$ expresses $\tilde{\mathscr{F}}$ and characterizes $v \tilde{\mathscr{F}}$.

Now we recall from [2] the declarations that characterize simulation equivalence and bisimulation equivalence.

Definition 5.3 Define $D_{\text {bisim }}=D_{\text {sim }} \wedge D_{\text {opsim }}$ and $D_{\text {simeq }}=v D_{\text {sim }} \wedge v D_{\text {opsim }}$.

Lemma $5.4 D_{\text {bisim }}$ characterizes $\sim_{\text {bisim }}$ and $D_{\text {simeq }}$ characterizes $\sim_{\text {sim }}$.

Proof $D_{\text {bisim }}$ does not contain nested fixed points and can therefore be interpreted directly over $\mathscr{V}_{0}=\mathscr{V}$. Now we proceed as follows:

$$
\Phi^{*}\left(\mathscr{F}_{\text {bisim }}\right)=\Phi^{*}\left(\mathscr{F}_{\text {sim }}\right) \cap \Phi^{*}\left(\mathscr{F}_{\text {opsim }}\right)=\mathscr{V}\left[\left[D_{\text {sim }}\right]\right] \cap \mathscr{V}\left[\left[D_{\text {opsim }}\right]\right]=\mathscr{V}\left[\left[D_{\text {sim }} \wedge D_{\text {opsim }}\right]\right]=\mathscr{V}\left[\left[D_{\text {bisim }}\right]\right] .
$$

To interpret $D_{\text {simeq }}$ we define $\Sigma_{1}=\{t t, f f\} \cup\left\{v D_{\text {sim }}(p) \mid p \in \mathbf{P}\right\}$ and $\Sigma_{2}=\Sigma_{1} \cup\left\{v D_{\text {opsim }}(p) \mid p \in \mathbf{P}\right\}$ and let $\mathscr{M}_{0}, \mathscr{M}_{1}, \mathscr{M}_{2}$ and $\mathscr{V}_{0}, \mathscr{V}_{1}$ be defined as before. Then $D_{\text {simeq }}: \mathbf{P} \rightarrow \mathscr{V}_{1}$. If we let $\mathscr{F}_{\text {simeq }}=v \mathscr{F}_{\text {sim }} \cap \boldsymbol{v} \mathscr{F}_{\text {opsim }}$, we get

$$
\begin{gathered}
\Phi^{*}\left(\mathscr{F}_{\text {simeq }}\right)=\Phi\left(v \mathscr{F}_{\text {sim }}\right) \cap \Phi\left(v \mathscr{F}_{\text {opsim }}=v \mathscr{V}_{1}\left[\left[D_{\text {sim }}\right]\right] \cap v \mathscr{V}_{1}\left[\left[D_{\text {opsim }}\right]\right]=\right. \\
\left.\mathscr{M}_{2}\left[\left[v D_{\text {sim }}\right]\right] \cap \mathscr{M}_{2}\left[v D_{\text {opsim }}\right]\right]=\mathscr{M}_{2}\left[\left[v D_{\text {sim }} \wedge v D_{\text {opsim }}\right]\right]=\mathscr{V}_{1}\left[\left[D_{\text {simeq }}\right]\right] .
\end{gathered}
$$

The result now follows from Cor.4.11.

Next we define the nested simulation preorders introduced in [5] by using the function $\mathscr{F}$. These definition involve nesting of fixed points and are defined recursively on the depth of the nesting. The 1-nested simulation $\sqsubseteq_{(1) \operatorname{sim}}$ is just the simulation preorder $\sqsubseteq_{\operatorname{sim}}$ as defined in Section 3 and the function $\mathscr{F}_{(1) \text { sim }}$ is therefore the function $\mathscr{F}$. As the preorder $\sqsubseteq_{(n+1) \text { sim }}$ depends on the inverse of the preorder $\sqsubseteq_{(n) \text { sim }}$, which we call $\sqsubseteq_{(n) o p s i m}$, we simultaneously define the nested simulations and their inverse in our recursive definition. The functions that define $\sqsubseteq_{(n) \operatorname{sim}}$ and $\sqsubseteq_{(n) \text { opsim }}$ are called $\mathscr{F}_{(n) \operatorname{sim}}$ and $\mathscr{F}_{(n) \text { opsim }}$ respectively.

\section{Definition 5.5 (Nested simulations)}
1. $\mathscr{F}_{(1) \text { sim }}=\mathscr{F}$ and $\sqsubseteq_{(1) \operatorname{sim}}=v \mathscr{F}_{(1) \text { sim }}$
2. $\mathscr{F}_{(1) \text { opsim }}=\widetilde{\mathscr{F}}$ and $\sqsubseteq_{(1) \text { opsim }}=\boldsymbol{v} \mathscr{F}_{(1) \text { opsim }}$,
3. $\mathscr{F}_{(n+1) \text { sim }}=\mathscr{F}_{(1) \text { sim }} \cap \boldsymbol{v} \mathscr{F}_{(n) \text { opsim }}$ and $\sqsubseteq_{(n+1) \text { sim }}=\boldsymbol{v} \mathscr{F}_{(n+1) \text { sim }}$.
4. $\mathscr{F}_{(n+1) \text { opsim }}=\mathscr{F}_{(1) \text { opsim }} \cap \boldsymbol{v} \mathscr{F}_{(n) \text { sim }}$ and $\sqsubseteq_{(n+1) \text { opsim }}=v \mathscr{F}_{(n+1) \text { opsim }}$.

We complete this note by defining a sequence of nested declarations and prove that they characterize the sequence of $n$-nested simulation preorders.

\section{Theorem 5.6}

1. $D_{(1) \text { sim }}=D$ expresses $\mathscr{F}_{(1) \text { sim }}$ and characterizes $\sqsubseteq_{(1) \text { sim }}$,

2. $D_{(1) \text { opsim }}=\widetilde{D}$ expresses $\mathscr{F}_{(1) \text { opsim }}$ and characterizes $\sqsubseteq_{(1) \text { opsim }}$,

3. $D_{(n+1) \text { sim }}=D_{(1) \text { sim }} \wedge v D_{(n) \text { opsim }}$ expresses $\mathscr{F}_{(n+1) \text { sim }}$ and characterizes $\sqsubseteq_{(n+1) \text { sim }}$, 
4. $D_{(n+1) \text { opsim }}=D_{(1) \text { opsim }} \wedge v D_{(n) \text { sim }}$ expresses $\mathscr{F}_{(n+1) \text { opsim }}$ and characterizes $\sqsubseteq_{(n+1) \text { opsim. }}$

Proof We prove the statements simultaneously by induction on $n$. First we note that $D_{1}, D_{2}, \ldots$, where $D_{2 i-2}=D_{(i) \operatorname{sim}}$ and $D_{2 i-1}=D_{(i) o p s i m}$ for $i \geq 1$ is a sequence of nested declarations. For the case $n=1$ we get from Lemma 5.2 that $\Phi^{*}\left(\mathscr{F}_{(1) \text { sim }}\right)=\mathscr{V}_{0}\left[\left[D_{(1) \text { sim }}\right]\right]$ and $\Phi^{*}\left(\mathscr{F}_{(1) \text { opsim }}\right)=\mathscr{V}_{1}\left[\left[D_{(1) \text { opsim }}\right]\right.$. Next assume that $\Phi^{*}\left(\mathscr{F}_{(n) \operatorname{sim}}\right)=\mathscr{V}_{2 n-2}\left[\left[D_{(n) \operatorname{sim}}\right]\right]$ and $\Phi^{*}\left(\mathscr{F}_{(n) \text { opsim }}\right)=\mathscr{V}_{2 n-1}\left[\left[D_{(n) \text { opsim }}\right]\right]$. To prove 3 . we proceed as follows:

$$
\begin{aligned}
& \Phi^{*}\left(\mathscr{F}_{(n+1) \operatorname{sim}}\right)=\Phi^{*}\left(\mathscr{F}_{(1) \operatorname{sim}}\right) \cap \Phi\left(\boldsymbol{v} \mathscr{F}_{(n) \text { opsim }}\right)=\mathscr{V}_{0}\left[\left[D_{(1) \operatorname{sim}}\right]\right] \cap \mathcal{V}_{2 n-2}\left[\left[D_{(n) \text { opsim }}\right]\right]= \\
& \mathscr{V}_{2 n-2}\left[\left[D_{(1) \operatorname{sim}} \wedge v D_{(n) \text { opsim }}\right]\right]=\mathscr{V}_{2 n}\left[\left[D_{(n+1) \operatorname{sim}}\right]\right] .
\end{aligned}
$$

Finally, to prove 4. we have:

$$
\begin{aligned}
& \Phi^{*}\left(\mathscr{F}_{(n+1) \text { opsim }}\right)=\Phi^{*}\left(\mathscr{F}_{(1) \text { opsem }}\right) \cap \Phi\left(\mathcal{V}_{(n) \text { sim }}\right)=\mathscr{V}_{1}\left[\left[D_{(1) \text { opsim }}\right] \cap \mathcal{V}_{2 n-1}\left[\left[D_{(n) \text { sim }}\right]\right]=\right. \\
& \mathscr{V}_{2 n-1}\left[\left[D_{(1) \text { opsim }} \wedge v D_{(n) \text { sim }}\right]\right]=\mathscr{V}_{2 n+1}\left[\left[D_{(n+1) \text { opsim }}\right]\right] .
\end{aligned}
$$

\section{References}

[1] L. Aceto, A. Ingolfsdottir, K.G. Larsen \& J. Srba (2007): Reactive Systems: Modelling, Specification and Verification. Cambridge University Press, doi $10.1017 /$ CBO9780511814105.

[2] L. Aceto, A. Ingolfsdottir, P. B. Levy \& J. Sack (2012): Characteristic Formulae for Fixed-Point Semantics: A General Framework. Mathematical Structures in Computer Science doi:10.4204/EPTCS.8.1. Special issue devoted to selected papers from EXPRESS 2009, Cambridge University Press.

[3] Jan Bergstra, Alban Ponse \& Scott A. Smolka, editors (2001): Handbook of Process Algebra. Elsevier.

[4] R. van Glabbeek (2001): The linear time-branching time spectrum. I. The semantics of concrete, sequential processes. In Bergstra et al. [3], pp. 3-99, doi 10.1016/B978-044482830-9/50019-9.

[5] Jan Friso Groote \& Frits W. Vaandrager (1992): Structured Operational Semantics and Bisimulation as a Congruence. Information and Computation 100(2), pp. 202-260, doi 10.1016/0890-5401(92)90013-6.

[6] M. Hennessy \& R. Milner (1985): Algebraic laws for nondeterminism and concurrency. Journal of the ACM 32(1), pp. 137-161, doi:10.1145/2455.2460.

[7] R.M. Keller (1976): Formal verification of parallel programs. Communications of the ACM 19(7), pp. 371-384, doi $10.1145 / 360248.360251$.

[8] Dexter Kozen (1983): Results on the Propositional mu-Calculus. Theoretical Computer Science 27, pp. 333-354, doi:10.1016/0304-3975(82)90125-6.

[9] Kim Guldstrand Larsen (1990): Proof Systems for Satisfiability in Hennessy-Milner Logic with Recursion. Theoretical Computer Science 72(2-3), pp. 265-288, doi 10.1016/0304-3975(90)90038-J.

[10] A. Tarski (1955): A Lattice-Theoretical Fixpoint Theorem and its Applications. Pacific Journal of Mathematics 5(2), pp. 285-309. Available at http://projecteuclid.org/euclid.pjm/1103044538 